Results: Baseline characteristic were significantly different between diagnoses (Table 1). In patients with RA, 60\% were able to work at baseline and $61 \%$ at 12 month, with PSA $71 \%$ and $74 \%$ resp., and with AS $72 \%$ and $77 \%$ resp. The main driver of improvement of WD was a change in the sick leave (RA $6 \% \rightarrow 2 \%$, PSA $7 \% \rightarrow 1 \%$, AS $9 \% \rightarrow 2 \%$ ). In univariate analyses, diagnosis of AS and younger age at start of a-TNF-th were predictive of improvement in WD. In multivariate analysis, only diagnosis of AS was significantly associated with improvement in WD.

Conclusions: These real life data from CZ show that for improvement of WD, a-TNF-th was most effective in patients with AS. This may be a sequel of disease specific factors or reimbursement policy.

Disclosure of Interest: None declared

DOI: 10.1136/annrheumdis-2017-eular.4056

\section{THU0622 PATIENT-CENTERED AGING BIOBANKS - A SURVEY ON PUBLIC PERCEPTIONS AND PATIENT CHOICE AMONG RHEUMATOLOGY OUTPATIENTS}

J.V. Cordeiro ${ }^{1}$, A. Fernandes ${ }^{2}$, C.D. Lopes $^{3}$, G. Victorino ${ }^{4}$, P. Lobato Faria ${ }^{1}$, M.J. Cercas ${ }^{4}$, J.C. Branco ${ }^{5}$, F.M. Pimentel-Santos ${ }^{3,5} .{ }^{1}$ Centro de Investigação em Saúde Pública (CISP), Escola Nacional de Saúde Pública; ${ }^{2}$ CICS.NOVA Centro Interdisciplinar de Ciências Sociais, Faculdade de Ciências Sociais e Humanas, NOVA University of Lisbon; ${ }^{3}$ Rheumatology, CHLO, Hospital Egas Moniz; ${ }^{4}$ NOVA Information Management School (NOVAIMS); ${ }^{5}$ CEDOC, NOVA Medical School/Faculdade Ciências Médicas, NOVA University of Lisbon, Lisboa, Portugal

Background: Biobanks for research (BBR) are organized repositories of biological materials and associated health information with enormous potential and value for scientific research. In consonance with increasing attention to healthy aging research, BBR specifically oriented to chronic diseases and aging populations have gathered heightened attention. Public perceptions and patient choices are key to design, develop and implement patient-centered BBR. Public awareness, education and involvement are confidence building and unequivocally lead to higher participation in scientific enterprises.

Objectives: To assess patient awareness, perception and choices regarding aging biobanking activities.

Methods: We developed and applied a standard anonymous questionnaire to rheumatology terciary outpatients, aged 50 or older, between March-October 2016. Demographic data and perceptions about biobanking were collected. Data analysis was performed using Stata $14^{\circledR}$ software.

Results: We obtained a total of 131 valid responses [age (min-max, 50-93), mean (64); sex ratio (M/F) (40/91, 44\%), education years (min-max, 0-20), mean (8.5)]. $69 \%$ of respondents did not know the specific term "biobank" but $57 \%$ were aware about the possibility of donating their biological material for research purposes. Furthermore, $77 \%$ of respondents indicated they were willing to contribute with their biological material to BBR, stating they had no particular preference whether these infrastructures were of private or public nature. However, they expressed a clear preference for these to be based at scientific research institutes (50\%), instead of hospitals (23\%), universities (16\%) or biotechnology companies $(7 \%)$. Moreover, respondents highlighted diferent requirements for their participation with anonymity $(31 \%)$ and confidentiality $(27 \%)$ ranking as top priorities. Most importantly, a majority of respondents $(70 \%)$ expressed their agreement with a biobank exclusively dedicated to the study of aging, considering that people of older ages have higher disease burdens and that such research infrastructures and practices expressed respect for the particular problems of the elderly (Figure).

Why dedicated Aging Biobanks?

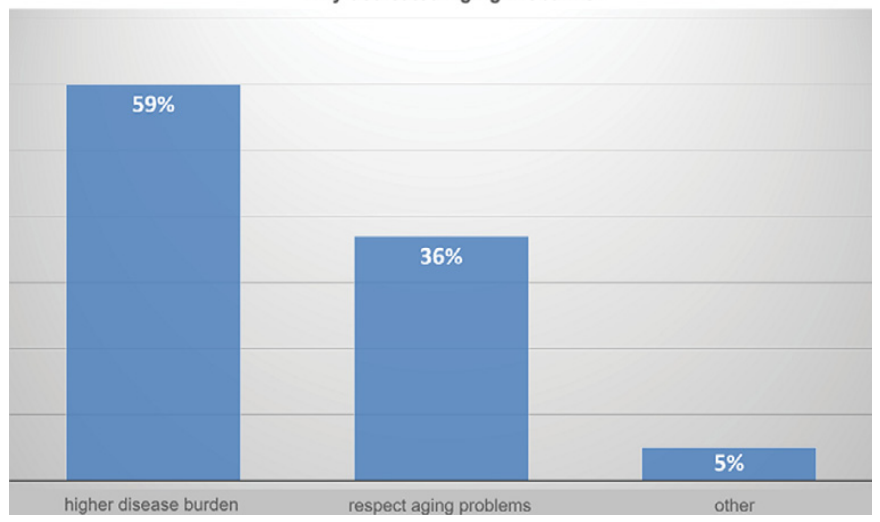

Conclusions: Our study constitutes a comprehensive assessment of public perceptions and patient choices regarding biobanks for aging research purposes among rheumatology outpatients. Although awareness is still suboptimal, BBR are highly regarded health infrastructures with enormous potential for further patient-centered development.

Disclosure of Interest: None declared

DOI: 10.1136/annrheumdis-2017-eular.6527

\section{THU0623 DECREASE IN THE TEMPORARY WORK INCAPACITY DUE TO MUSCULOSKELETIC DISEASES: UTILITY OF A PREVENTIVE PROGRAM IN VALENCIA-LA FE HEALTH AREA}

J.E. Oller Rodríguez ${ }^{1}$, M.T. Fuente Goig ${ }^{2}$, F. Ortiz Sanjuan ${ }^{1}$, J. Ivorra Cortés ${ }^{1}$, E. Grau García ${ }^{1}$, E. Vicens Bernabéu ${ }^{1}$, E. Labrador Sánchez ${ }^{1}$, K.R. Arévalo Ruales $^{1}$, J. Fragio Gil ${ }^{1}$, R. González Mazario ${ }^{1}$, C.P. Alcañiz Escandell ${ }^{1}$, I. Cánovas Olmos ${ }^{1}$, I. Chalmeta Verdejo ${ }^{1}$, C.M. Feced Olmos ${ }^{1}$, L. González Puig ${ }^{1}$, I. Martínez Cordellat ${ }^{1}$, C. Nájera Herranz ${ }^{1}$, R. Negueroles Albuixech ${ }^{1}$, J. Andani Cervera ${ }^{2}$, J.A. Román Ivorra ${ }^{1}$ on behalf of Rheumatology Service. ${ }^{1}$ Rheumatology; ${ }^{2}$ Service of Prevention of Occupational Hazards, Hospital Universitari I Politècnic la Fe, Valencia, Spain

Background: Musculoskeletal diseases are nowadays a frequent cause of temporary work incapacity (TWI). The implementation of specific programs for the care of these patients can be an important cornerstone in the resolution of these TWI processes.

Objectives: Our aim is to analyze the usefulness of an Early Intervention Program $(E I P)$ in patients with TWI of musculoskeletal origin.

Methods: Case-control study, including patients from HUP La Fe area, which were referred from Primary Care since April 2012 to April 2016 to our Early intervention program (EIP) addressed to patients with TWI of musculoskeletal origin. The patients were evaluated in our consultation within a maximum of 15 days after the start of TWI. The intervention consisted in an evaluation, including complementary tests if necessary, diagnosis and treatment by the rheumatologist in consultation. The patient was followed in consultation continuously until discharge. Patients whose disabilities were of traumatic or surgical origin were excluded. A comparative study was carried out against a control group of patients with ILT of musculoskeletal origin.

Results: A total of 666 patients were recruited; 508 (76.3\%) belonged to EIP group (46.1\% male; $53.9 \%$ female) and 158 patients $(23.7 \%)$ belonged to the control group ( $44.3 \%$ male; $55.7 \%$ female).

The average age was $47,6 \pm 10.5$ years for the EIP ones and $46.7 \pm 10$ years in the control group.

The most frequent diagnoses were low back pain $(23.3 \%)$, neck pain $(18 \%)$ y lumbosciatica (12.3\%). In EIP group, $100 \%$ of the patients received medical treatment, a $54.5 \%$ received instructions for doings physical therapy at home, an ultrasound scan was performed to the $26,4 \%$ of the patients while the $19.9 \%$ received at least one local infiltration. The longest TWI corresponded to knee meniscopathy (203 days), painful shoulder syndrome (173 days) and lumbosciatica (170.5 days). No statistically significant differences were found between the duration of the TWI attending to sex, age group, labour activity or diagnose. However, a significant association was found between TWI duration and the delay since the start of the symptons to the referral from Primary Care to our consultation, specially within the firdt 10 days $(p=0.04)$. Furthermore, TWI duration was significantly shorter in the EIP group patients than in the control group patients $(137.4 \pm 132.3$ days vs $194.7 \pm 143.1$ days; $p<0,001)$. The period before relapse was longer in the EIP group, although the differences did not reach significance. In addition, an inverse relation was found between age and time to relapse $(p=0,01)$

Conclusions: The establishment of an early intervention program specifically addressed to patients with temporary work incapacity of musculoskeletal origin shortens the duration of this situation, allowing the patient to rejoin his work activity, with the resulting cost savings related to work incapacity.

Disclosure of Interest: None declared

DOI: 10.1136/annrheumdis-2017-eular.5486

\section{THU0624 ASESSMENT OF PSYCHIC EXPERIENCES IN PATIENTS WITH RHEUMATIC DISEASES}

J.L. Prados-Ojeda ${ }^{1}$, J.L. Garrido-Castro ${ }^{2}$, R. Segura-Ruiz ${ }^{1}$,

C. González-Navas ${ }^{2}$, P. Font-Ugalde ${ }^{2}$, E. Collantes-Estevez ${ }^{2}$,

A. Escudero-Contreras ${ }^{1}$. ${ }^{1}$ Reina Sofia University Hospital; ${ }^{2}$ Maimonides Institute for Biomedical Research of Cordoba, Cordoba, Spain

Background: There are several studies that report psychiatric comorbidity in patients with rheumatic disease, mainly the presence of mood disorders. Some of them describe non-affective psychosis related with inflammatory processes. Population with chronic inflammatory disease could present subclinical psychotic experiences that can interfere in the patient's functional status. The Community Assessment of Psychic Experiences (CAPE) scale is a validated and widely used tool for the evaluation of these experiences in the general population.

Objectives: To identify the presence of psychic experiences in different populations with a diagnosis of rheumatic disease, and to compare it with a sample of healthy subjects.

Methods: 124 subjects completed surveys including SF-12 and CAPE questionnaires, as well as other demographic and behavioral variables. Among them, 70 had Spondylarthritis (SpA) (age 44.3 \pm 13 years, 62\% female), 23 rheumatoid arthritis (RA) (age $51.2 \pm 13$ years, $82 \%$ female) and the rest were individuals without rheumatic diseases ( $47.6 \pm 12$ years, $58 \%$ female).

Results: Results of the SF-12 test in their mental and physical domains, and the CAPE questionnaire in their dimensions (positive, negative, depressive and total symptoms) are shown in the table, expressed as mean value (SD) and in the graph expressed as density histograms with mean values. Significant statistical 